\title{
A Pilot Trial of Ambulatory Monitoring of Gastric Motility Using a Modified Magnetic Capsule Endoscope
}

\author{
Hee Man Kim, ${ }^{1 *}$ Ja Sung Choi ${ }^{2}$ and Jae Hee Cho ${ }^{3}$ \\ ${ }^{1}$ Division of Gastroenterology and Hepatology, Department of Internal Medicine, Yonsei University Wonju College of Medicine, Wonju, \\ Gangwon-do, Korea; '2 Division of Gastroenterology, Department of Internal Medicine, Myongii Hospital, Goyang, Gyeonggi-do, Korea; and \\ ${ }^{3}$ Division of Gastroenterology, Department of Internal Medicine, Gachon University Gil Medical Center, Incheon, Korea
}

The magnetic capsule endoscope has been modified to be fixed inside the stomach and to monitor the gastric motility. This pilot trial was designed to investigate the feasibility of the magnetic capsule endoscope for monitoring gastric motility. The magnetic capsule endoscope was swallowed by the healthy volunteer and maneuvered by the external magnet on his abdomen surface inside the stomach. The magnetic capsule endoscope transmitted image of gastric peristalsis. This simple trial suggested that the real-time ambulatory monitoring of gastric motility should be feasible by using the magnetic capsule endoscope.

\section{(J Neurogastroenterol Motil 2014;20:261-264)}

Key Words

Capsule endoscopy; Monitoring, ambulatory; Motility; Stomach

\section{Introduction}

Several techniques have been developed to assess gastric motility and gastric emptying. ${ }^{1}$ The cutaneous electrograstrography (EGG) represents the myoelectrical activity of the stomach, and is interpreted into imaginary gastric movement. ${ }^{1,2}$ Scintigraphy using radionuclide luminescence imaging provides visual movement of the stomach, such as gastric emptying, that is rough and disconnected. ${ }^{3}$ These 2 techniques represent indirect images of gastric motility.

Wireless capsule endoscopy is mainly used for identifying small bowel diseases. The current capsule endoscope goes through the gastrointestinal (GI) tract passively by gravity and peristalsis. If it would be fixed or located in the stomach for some time, the capsule endoscope could monitor gastric motility continuously, not passing through lower part of the GI tract. A magnetic capsule endoscope had been developed for the fixation in the stomach, and it was investigated to determine whether a modified magnetic capsule endoscope was feasible for monitoring

Received: December 4, 2013 Revised: February 19, 2014 Accepted: March 4, 2014

(c) This is an Open Access article distributed under the terms of the Creative Commons Attribution Non-Commercial License (http://creativecommons. org/licenses/by-nc/3.0) which permits unrestricted non-commercial use, distribution, and reproduction in any medium, provided the original work is properly cited.

*Correspondence: Hee Man Kim, MD, PhD

Division of Gastroenterology and Hepatology, Department of Internal Medicine, Yonsei University Wonju College of Medicine, 20 Ilsan-ro, Wonju, Gangwon-do 220-701, Korea

Tel: +82-33-741-1229, Fax: +82-31-741-1228, E-mail: loverkorea2009@gmail.com

Financial support: This study was supported by a grant from the Gachon University Gil Medical Center (Grant No. 2013-35).

Conflicts of interest: None.

Author contributions: Hee Man Kim: conception and design, or analysis and interpretation of data; Ja Sung Choi: drafting the article; Jae Hee Cho: revising it critically for important intellectual content. 


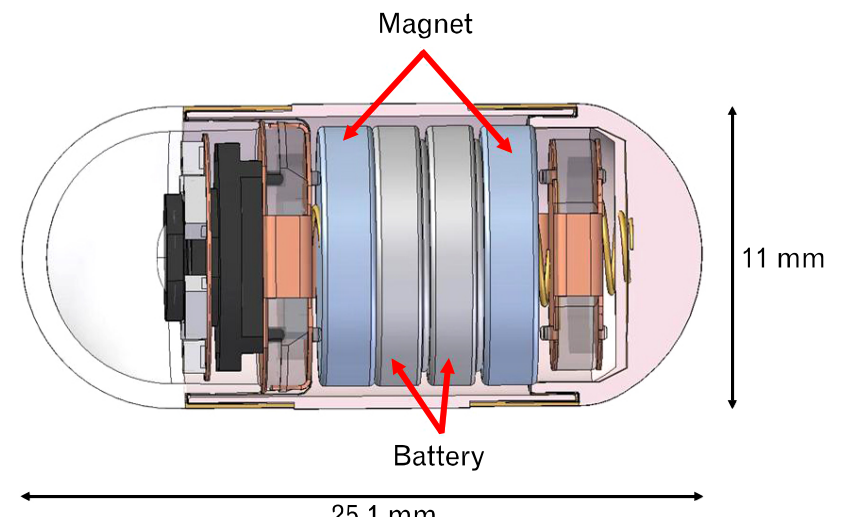

$25.1 \mathrm{~mm}$

Figure 1. The magnetic MiroCam is a modified capsule endoscope with 2 small magnetic disks incorporated. This image was provided by IntroMedic Company.

real-time and continuous gastric motility inside the stomach. In this report, we described the first experience of intra-gastric monitoring of gastric motility using a magnetic capsule endoscope.

\section{Case Report}

For development of a magnet capsule endoscope, 2 small permanent magnetic disks are incorporated into MiroCam (IntroMedic, Seoul, Korea), a capsule endoscope, using a novel communication mechanism that is not interfered by magnetic force (Fig. 1). ${ }^{4}$ This modified MiroCam was provided by IntroMedic Company. An external round magnet controls the movement of the magnetic MiroCam inside the stomach.

A healthy volunteer swallowed the magnetic MiroCam after the electrodes were attached on his abdomen surface. The images captured by the magnetic MiroCam were shown on the real-time viewer program. The external magnet on the abdominal surface maneuvered the magnetic MiroCam inside the stomach and moved it to the greater curvature of the lower body in order to observe motility around gastric angle. At the intended location, the external magnet was fixed on the volunteer's abdomen using an abdomen belt with 16 small magnets arranged in a regular fashion (Fig. 2A and 2B). Daily activity was allowed to the subject (Fig. 2C). After 30 minutes, the belt and external magnet were removed. The transmitted images from the magnetic MiroCam fixed inside the stomach showed continuous gastric peristalsis around the gastric angle (Fig. 3 and Supplementary Video). Our short successful experience suggests that the magnetic capsule endoscopy may be available to monitor gastric motility inside the stomach.
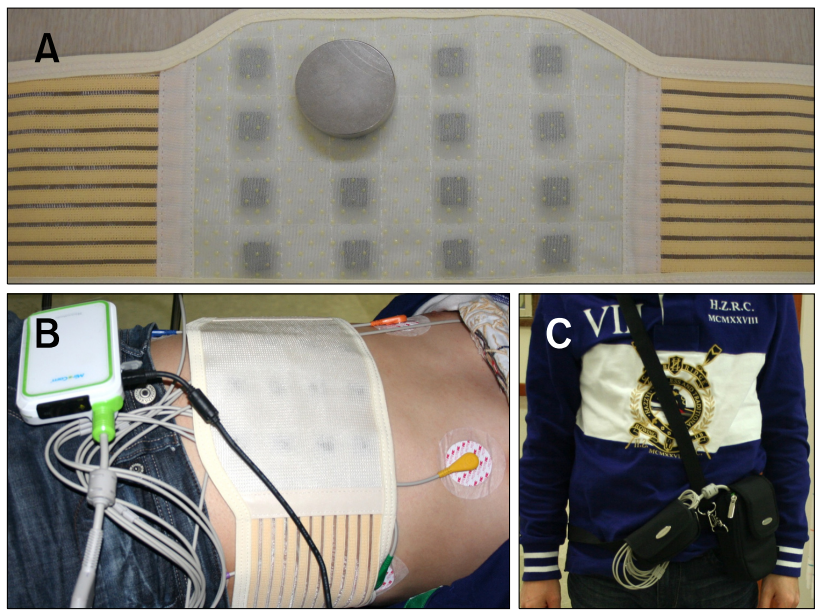

Figure 2. Preparation for ambulatory monitoring of gastric motility. (A) An external round magnet is located on the abdominal belt with 16 small magnets. (B) Abdominal belt fixed the external magnet on the subject's abdominal surface. (C) The subject was ambulatory.

\section{Discussion}

This is a pilot trial to test the possibility to monitor real-time gastric motility inside the stomach. The gastric motility can be observed even through esophagogastroduodenoscopy when it is inserted into the stomach, but the gastric motility is not physiologic because the large amount of air is inflated into the stomach, and because the long shaft of endoscope is positioned on mouth, esophagus and stomach simultaneously and continuously. Also it cannot be digitized as indicators presenting figures. Wireless capsule endoscopy can visualize inside of the stomach without air inflation and a long shaft, and its size is so small that it minimally affects the stomach motility, as compared with esophagogastroduodenoscopy. Although this trial is so primitive that the information is only the feasibility to monitor gastric motility using a modified capsule endoscopy, this experience may help with investigation on gastric motility disorders. The various types of magnet capsule endoscopes for the stomach have been tried to screen gastric diseases as a substitute for conventional esophagogastroduodenoscopy. ${ }^{5-7}$ However, in our trial, magnetic capsule endoscopy brought focus on gastric motility. Our simple trial and outcome indicate the possibility of ambulatory direct monitoring of gastric movement. The real-time monitoring of gastric motility will help the investigation of gastric motility disorders.

The cutaneous EGG is a non-invasive technique recording myoelectric activity of the stomach on the abdominal skin, and used to identify the pathophysiology of the functional GI disorders. ${ }^{8}$ However, the cutaneous EGG does not measure gas- 

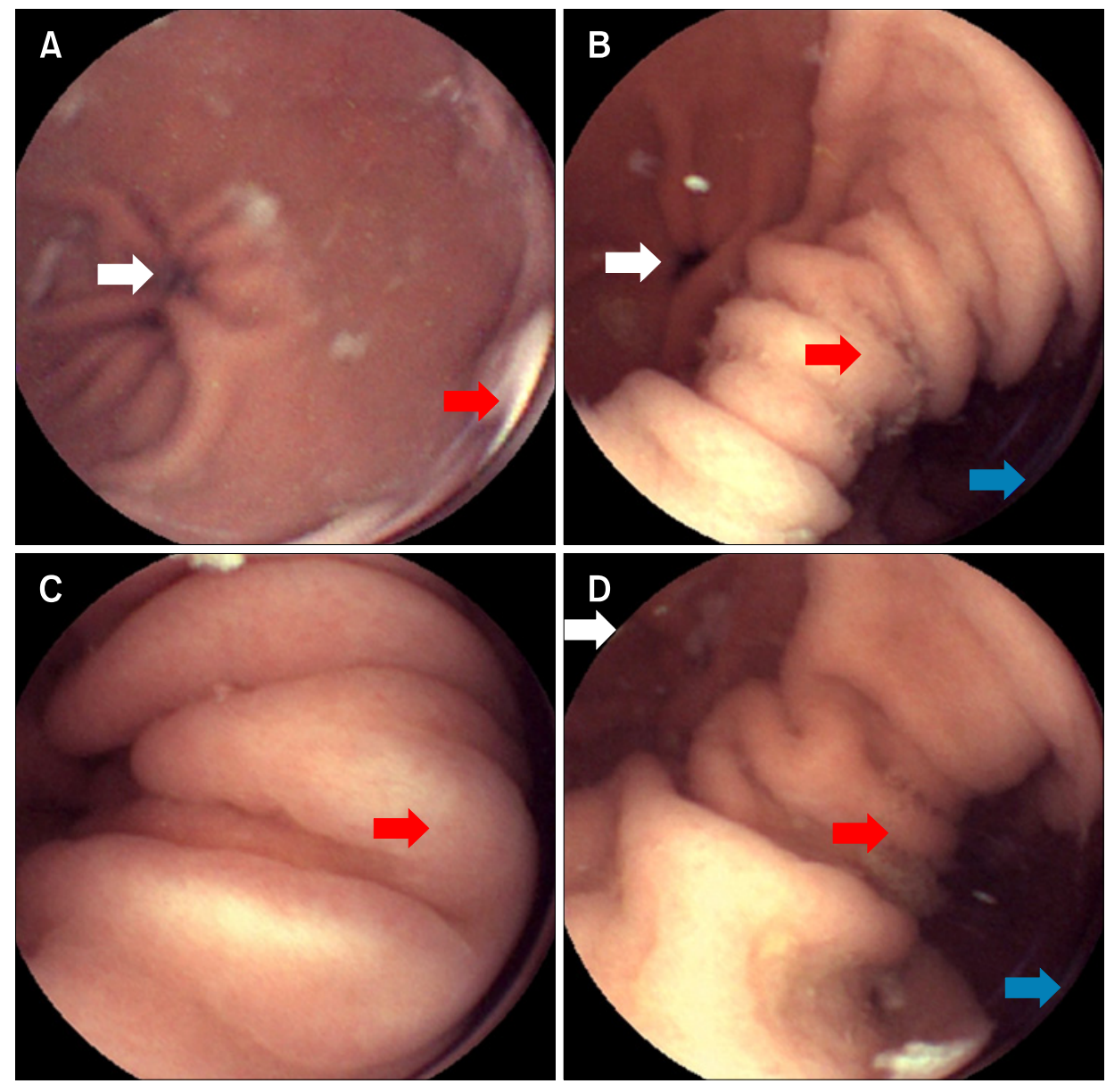

Figure 3. The magnetic MiroCam fixed inside the stomach transmitted real-time images showing gastric peristalsis moving to the antrum (A) from the angle (B-D). White arrow is pylorus, red arrow is angle and blue arrow is greater curvature of the lower gastric body.

tric emptying nor gastric contraction, ${ }^{2}$ and it records gastric slow waves determining frequency and propagation of antral contractions. The correlation between EGG and gastric emptying remains controversial. ${ }^{9}$ Also, it is difficult to reliably record and analyze the signal. ${ }^{9}$ A magnetic capsule endoscopy may supplement the cutaneous EGG if two techniques would be combined by synchronizing myoelectrical activity and real-time gastric motility. Gastric-emptying scintigraphy is a standard technique to measure gastric emptying. ${ }^{10}$ It is a non-invasive and quantitative method, but the measurement is rough and disconnected. ${ }^{3}$ A magnetic capsule endoscopy may provide continuous visual gastric motility and qualitative aspects of the motility.

This report is the first step to develop the novel technique for intra-gastric monitoring of the gastric motility, although there are lots of problems to be solved: the validation should be performed; the quantitative measurement of gastric motility for determining normal motility should be further investigated also; and the cost-effectiveness should be evaluated.

In summary, a magnetic capsule endoscopy provided re- al-time images of intra-gastric motility. The combination of the cutaneous EGG and a magnetic capsule endoscopy will be a novel technique to investigate gastric motility disorders.

\section{Supplementary Material}

Note: To access the supplementary video mentioned in this article, visit the online version of Journal of Neurogastroenterology and Motility at http://www.jnmjournal.org/, and at doi: http://dx.doi.org/10.5056/jnm.2014.20.2.261.

\section{References}

1. Pohle T, Domschke W. Gastric function measurements in drug development. Br J Clin Pharmacol 2003;56:156-164.

2. Yin J, Chen JD. Electrogastrography: methodology, validation and applications. J Neurogastroenterol Motil 2013;19:5-17.

3. Olausson EA, Brock C, Drewes AM, et al. Measurement of gastric emptying by radiopaque markers in patients with diabetes: correlation with scintigraphy and upper gastrointestinal symptoms. Neurogastroenterol Motil 2013;25:e224-e232. 
4. Kim HM, Kim YJ, Kim HJ, et al. A pilot study of sequential capsule endoscopy using MiroCam and PillCam SB devices with different transmission technologies. Gut Liver 2010;4:192-200.

5. ASGE Technology Committee, Gottlieb KT, Banerjee S, et al. Magnets in the GI tract. Gastrointest Endosc 2013;78:561-567.

6. Keller J, Fibbe C, Volke F, et al. Inspection of the human stomach using remote-controlled capsule endoscopy: a feasibility study in healthy volunteers (with videos). Gastrointest Endosc 2011;73:2228.

7. Rey JF, Ogata H, Hosoe N, et al. Feasibility of stomach exploration with a guided capsule endoscope. Endoscopy 2010;42:541-545.

8. Chang FY. Electrogastrography: basic knowledge, recording, processing and its clinical applications. J Gastroenterol Hepatol 2005;20 502-516.

9. Riezzo G, Russo F, Indrio F. Electrogastrography in adults and children: the strength, pitfalls, and clinical significance of the cutaneous recording of the gastric electrical activity. Biomed Res Int 2013; 2013:282757.

10. Tang DM, Friedenberg FK. Gastroparesis: approach, diagnostic evaluation, and management. Dis Mon 2011;57:74-101. 